\title{
Charge breeding ions for nuclear astrophysics experiments at ISOLDE
}

\section{Marie-Jeanne ${ }^{* a, b, c}$ and P. Delahaye ${ }^{a}$}

${ }^{a}$ CERN, Physics Department, 1211 Geneva 23, Switzerland

${ }^{b}$ LPSC Grenoble, 38026 Grenoble Cedex, France

${ }^{c}$ Université Joseph Fourier, 38041 Grenoble Cedex 9, France

E-mail: melanie.marie-jeanne@cern.ch, pierre.delahaye@cern.ch

\begin{abstract}
At ISOLDE, a PHOENIX ECR charge breeder is being tested for the investigation of the $1^{+} \rightarrow$ $\mathrm{n}^{+}$scenario for the next generation ISOL-type facilities. As a side objective, the possible physics experiments with intense multicharged radioactive ion beams are being investigated. Especially the use of the ECR charge breeder in combination with a high voltage platform would permit an acceleration of the radioactive ions produced at ISOLDE to total energies up to a few $\mathrm{MeV}$. This opens up possibilities for astrophysics experiments such as different studies of low energy radiative capture reactions. Here, the current developments of the ECR charge breeder at ISOLDE are presented, in view of its future possible use for these particular physics experiments.
\end{abstract}

International Symposium on Nuclear Astrophysics - Nuclei in the Cosmos - IX

June 25-30 2006

CERN, Geneva, Switzerland

\footnotetext{
* Speaker.
} 


\section{Introduction}

At ISOLDE, CERN, an on-line test bench is dedicated to charge breeding experiments with the Daresbury ECR Phoenix Booster. Due to the high diversity of exotic nuclides produced far from stability, the optimization of the charge breeder performances is challenging. Therefore, the Phoenix Booster undergoes several technical developments to meet the requirements for rapid, efficient and high $\mathrm{A} / \mathrm{q}$ charge breeding.

\section{Status of the Phoenix Booster charge breeder}

The Phoenix booster is installed on-line, on the heavy-mass parasitic beamline (GHM) of the General Purpose Separator (GPS) [1]. Singly charged ions produced by ISOLDE are continuously injected in the plasma chamber. There, free electrons gyrate around the magnetic field lines with the cyclotron frequency $\omega_{c}$. When microwave radiation of the same frequency propagates into such a region, the electrons are resonantly accelerated or decelerated depending on the phase of their transversal velocity component with respect to the electric field vector. Thus, the electron cyclotron resonance condition is the following:

$$
\omega_{h f}=\omega_{c}=\frac{e B}{m}
$$

where e and $\mathrm{m}$ respectively denote the charge and mass of the electron, and $\mathrm{B}$ the magnetic field intensity.

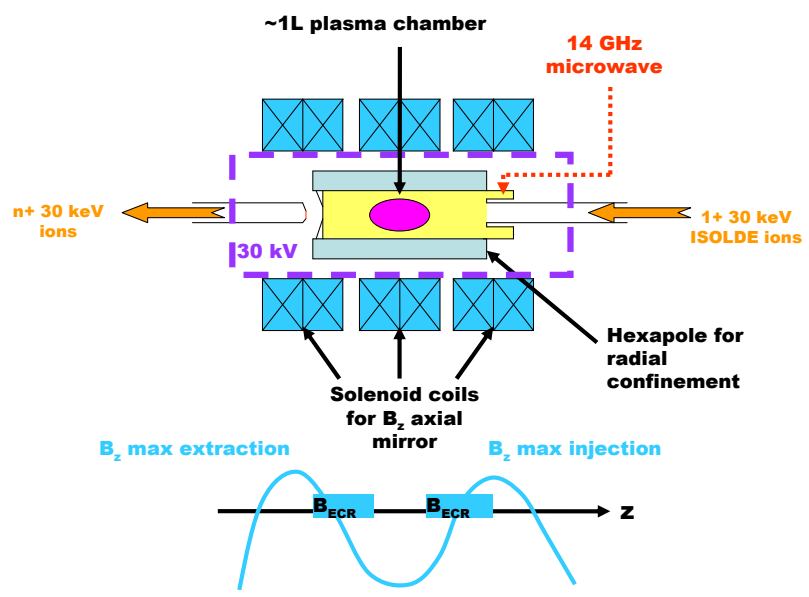

Figure 1: Ion motion inside the ECR charge breeder: the 1+ ions are stopped and then ionized in the plasma. The plasma is trapped in a configuration where the axial magnetic field B shows a minimum.

The electrons of the plasma are confined in a so-called minimum-B-structure described by Fig. 1. A closed surface is created where the electron cyclotron resonance condition is fulfilled. The high mirror ratio of the magnetic field leads to long confinement times for the plasma electrons. They can pass thousands times the resonance region, stochastically gaining energy from a high frequency electromagnetic wave and ionizing plasma ions to high charge states via stepwise 
ionization. After extraction, a $90^{\circ}$ bending magnet allows a selection in $\sqrt{A / q}$, with $\mathrm{A}$ the atomic mass and $\mathrm{q}$ the ion charge state, before ions are collected in a Faraday cup at the end of the line. The latest on-line charge breeding results of the Phoenix booster are described in [2]. It is currently able to charge breed a wide range of nuclides for $\mathrm{A} / \mathrm{q}$ values ranging from 4 to 8 , either in continuous or in pulsed mode. Efficiencies for the injected elements are similar to the ones measured for the REX beam preparation stage [3].

\section{The project of separation in mass and in energy}

Recent developments on the ECR charge breeder aim at more flexibility of operation, and at a better purity of the beam. On the first hand, the upgrade of the ECR potential platform from $30 \mathrm{kV}$ to $60 \mathrm{kV}$ will allow to handle more beams among the available ISOLDE production. On the other hand, the improvement of the vacuum and the installation of a two-step separator at the ejection side will contribute to a better purity of the beam.

The latter point requires a careful study. Previous measurements have shown the existence of a $10 \mathrm{nA}$ background in the A/q range from 3 to 7 (see Fig. 2). This level is believed to be caused by charge recombinations inducing energy spread on the A/q values. Therefore energy selection is to be added, after the charge breeder and before A/q separation. A design of the type of the Nier-spectrometer design was already set up on the REX-ISOLDE facility [4] and is chosen for our purpose.

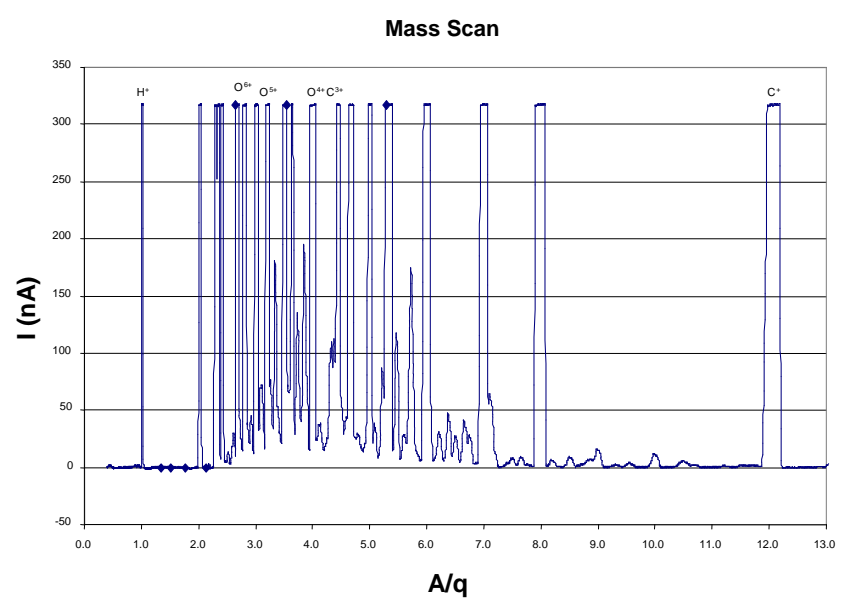

Figure 2: Mass spectrum in continuous mode with a 10nA background

Simulations were done to design the new isotopic separator setup with elements provided after the dismantlement of the AMS facility injector [5] in Lund. The energy and mass separator is constituted of two analyzers: the electrostatic analyzer reduces interference of energy tails from nearby masses and the magnetic analyzer provides the $\mathrm{A} / \mathrm{q}$ selection. The electrostatic analyzer has a bending radius of $435 \mathrm{~mm}$ and a bending angle of $90^{\circ}$. The analyzer plates are spherical in the transversal plane of the beam and provide a double focusing both in the $\mathrm{X}$ and $\mathrm{Y}$ directions. The magnetic analyzer has a bending angle of $90^{\circ}$ and a uniform field in the gap. A pole edges rotation 
of $28.2^{\circ}$ provides a double focusing effect here too. Therefore, stigmatic imaging can be performed for one isotope through the whole analysis system. An optimization routine was performed with the COSY INFINITY software [6] [7] to evaluate the relative positions of the analyzers and of the possible focusing elements. The ions distribution through the computed transfer matrixes was calculated under Matlab to estimate the resolving power for $\mathrm{A} / \mathrm{q}$ values under 4. A good balance between transmission and resolution resulted in a preliminary energy resolving power $\frac{E}{\Delta E} \approx 160$ and a preliminary mass resolving power $\frac{m}{\Delta m} \approx 140$ (see Fig. 3). Two quadrupole triplets were needed in the final setup for an optimized control of the beam before and after the analyzers (see Fig. 4).

(A)

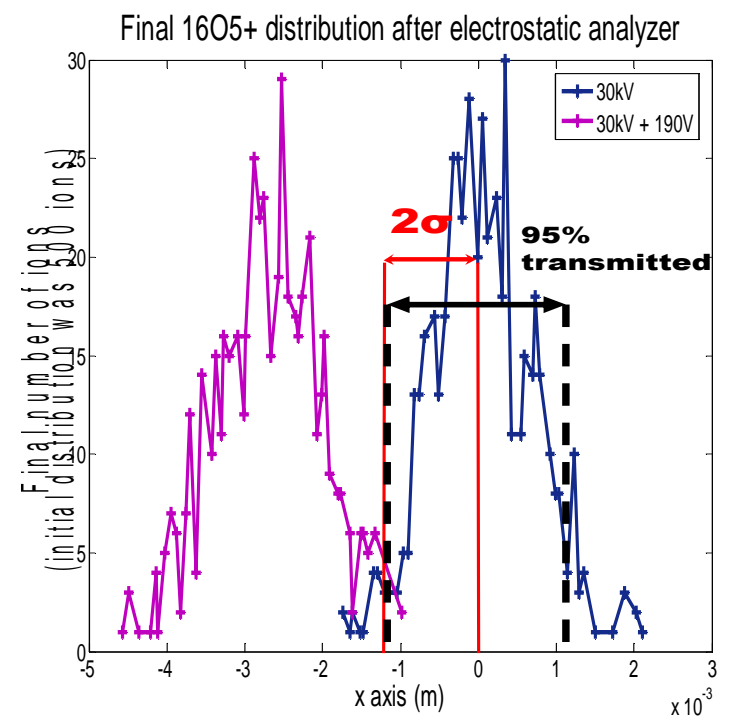

(B)

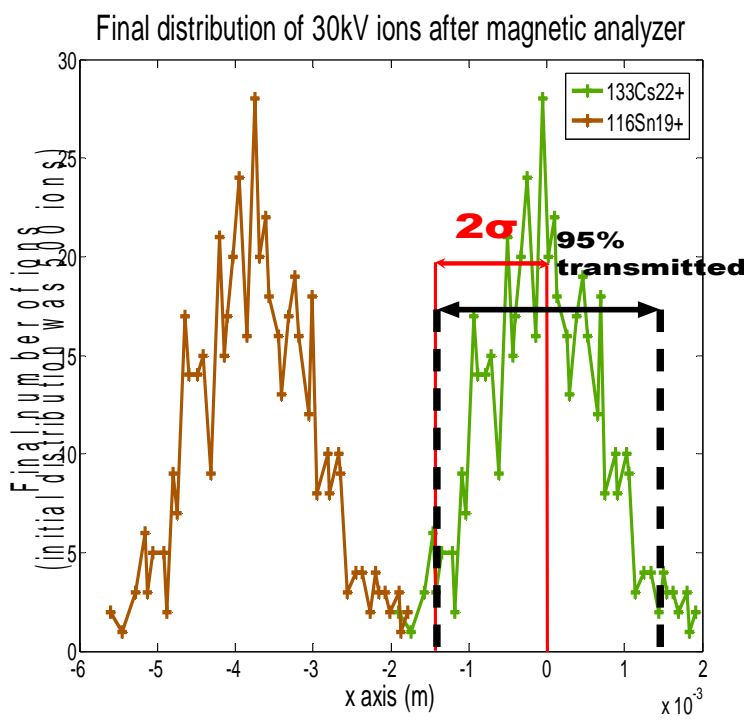

Figure 3: Simulations of $(A){ }^{16} \mathrm{O}^{5+}$ ions with different energies and of $(B){ }^{16} \mathrm{O}^{5+}$ and ${ }^{17} \mathrm{O}^{5+}$ ions accelerated at the same potential through the isotopic separator. The beam has an emittance of $30 \pi . \mathrm{mm}$. mrad and a potential energy of $30 \mathrm{keV}$ per charge.

\section{Outlook}

The charge breeding of radioactive ions in ion sources is now commonly used for an efficient post-acceleration of the ISOL-type beams in LINAC or cyclotrons. Besides this application, it presents many advantages. Especially, it allows the production of very pure beams by suppressing the contaminants with molecular sidebands [8] or charge state distributions [9]. Moreover, it can provide a full electrostatic post-acceleration with the use of a high voltage cage [10], filling the gap between the energies available after the mass separation at typical ISOL-type facilities, usually from $30 \mathrm{kV}$ to $60 \mathrm{kV}$, and the first energies accessible after a RFQ cavity, of the order of a few hundreds of kev/u.

This property is of interest in perspective of "low-energy" nuclear astrophysics experiments. For example, radiative proton and alpha capture reactions play an important role in the synthesis of elements heavier than oxygen. In facilities such as DRAGON (TRIUMF, Vancouver), absolute rates 
(A)

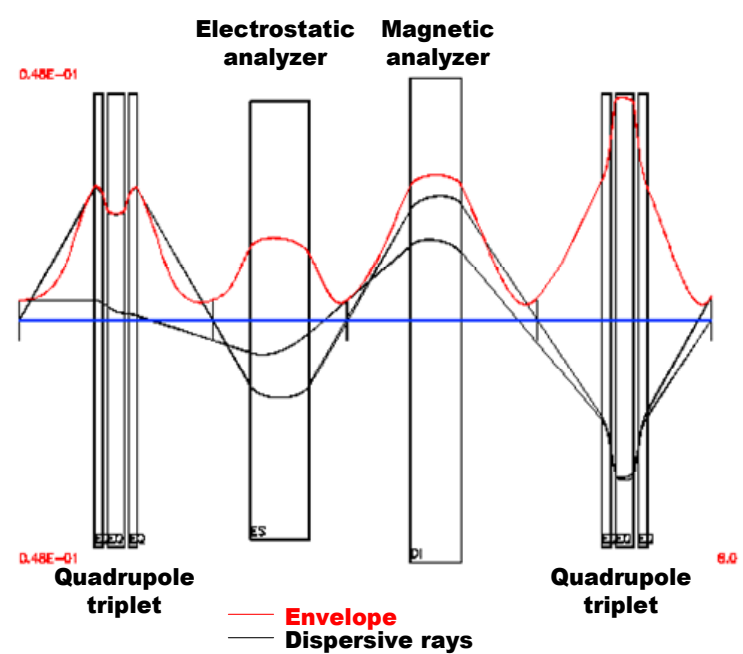

(B)

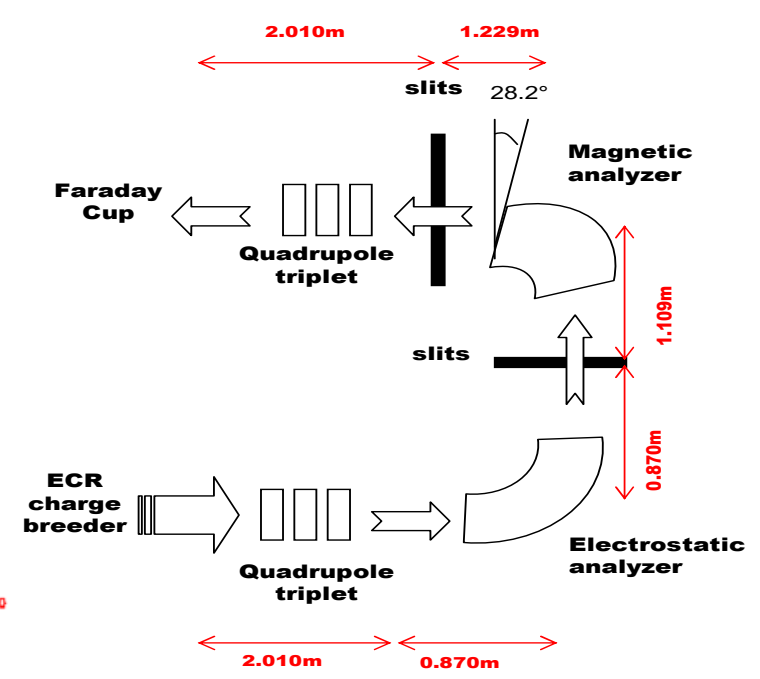

Figure 4: (A) Simulations of the beam envelope through the isotopic separator in the dispersive plane with COSY INFINITY. (B) Preliminary sketch of the separator setup with the calculated relative distances.

for these reactions can be measured in the energy range of 0.15 to $1.5 \mathrm{MeV} / \mathrm{u}$ [11]. At ISOLDE, a simple high-voltage platform setup after the ECR charge breeder would provide an alternative way of accelerating intense beams for the study of low-lying resonances. As an example, the case of the ${ }^{8} \mathrm{Li}(\alpha, n){ }^{11} \mathrm{~B}$ reaction [12] was investigated. With a production of about $10^{8}$ singly charged ${ }^{8} \mathrm{Li}$ per second from a Ta foil target coupled with a tungsten surface ionizer, and an efficiency per charge state of about $10 \%$ from the charge breeder, a beam of about $10^{7}{ }^{8} \mathrm{Li}^{3+}$ ions would be available. The first resonant states in ${ }^{12} \mathrm{~B}$ lying at $10.115 \mathrm{MeV}$ and $10.217 \mathrm{MeV}$ could then be accessed with good statistics by using respective electrostatic acceleration voltages of $-304 \mathrm{kV}$ and $-607 \mathrm{kV}$.

\section{References}

[1] E. Kugler, The ISOLDE facility, Hyp. Int. 129 (2000), 23

[2] P. Delahaye et al., Recent results with the Phoenix Booster at ISOLDE, Rev. Sci. Instrum. 77, $03 \mathrm{~B} 105$ (2006)

[3] F. Wenander et al., The REX-ISOLDE charge breeder as an operational machine, Rev. Sci. Instrum. 77, 03B104 (2006)

[4] D. Habs et al., The q/m-separator for REX-ISOLDE, Nucl. Instr. and Meth. B 126, 218-223 (1997)

[5] R. Hellborg et al., A high resolution AMS-injector for the Pelletron in Lund, Pramana, J. Phys., 59:6 1061-1073 (2002)

[6] M. Berz et al., COSY 5.0 - The Fifth Order Code for Corpuscular Optical Systems, Nucl. Instr. and Meth. A 258, 402-406 (1987) 
[7] K. Makino et al., COSY INFINITY Version 9, Nucl. Instr. and Meth. A 558, 346-350 (2005)

[8] P. Delahaye et al., Recent developments of the radioactive beam preparation at REX-ISOLDE, Eur. Phys. J. A 25, s01, 739-741 (2005)

[9] L. Weissman et al., $\beta$ decay of ${ }^{49,50} A r$, PRC 67 (2003) 054314, $\beta$ decay of ${ }^{47} A r$, PRC 70 (2004) 024304

[10] M. Hass, Possible configuration for a high-charge-state high-voltage platform, CERN internal report, AB-Note-2004-034-OP (2004)

[11] S. Engel, Commissioning and operation of DRAGON, NIM B 204 (2003) 154-158

[12] S. Kubono et al., Key resonant states in the ${ }^{8} \mathrm{Li}(\alpha, n){ }^{11}$ B reaction in the inhomogeneous big bang models, Z. Phys. A 338 (1991) 459-460 